\title{
SpoTyping: fast and accurate in silico Mycobacterium spoligotyping from sequence reads
}

\author{
Eryu Xia ${ }^{1}$, Yik-Ying Teo $1,2,3,4,5$ and Rick Twee-Hee Ong ${ }^{2 *}$
}

\begin{abstract}
SpoTyping is a fast and accurate program for in silico spoligotyping of Mycobacterium tuberculosis isolates from nextgeneration sequencing reads. This novel method achieves high accuracy for reads of both uniform and varying lengths, and is about 20 to 40 times faster than SpolPred. SpoTyping also integrates the function of producing a report summarizing associated epidemiological data from a global database of all isolates having the same spoligotype. SpoTyping is freely available at: https://github.com/xiaeryu/SpoTyping-v2.0.
\end{abstract}

\section{Background}

Tuberculosis (TB), caused mainly by Mycobacterium tuberculosis $(M t b)$, is a top infectious disease killer around the world and remains an acute international health problem, resulting in an estimated 9.6 million new cases and 1.5 million deaths globally in 2014 [1]. The global emergence and spread of drug-resistant TB have compounded the difficulty of treating and eradicating this disease.

Spoligotyping (spacer oligonucleotide typing) is a widely used genotyping method for $M t b$, which exploits the genetic diversity in the clustered regularly interspersed short palindromic repeats (CRISPR) locus, which is also known as the direct repeat (DR) locus in $M t b$ genome [2]. Each DR region consists of several copies of the 36 bp DR sequence, which are interspersed with $34 \mathrm{bp}$ to $41 \mathrm{bp}$ non-repetitive spacers [3]. A set of 43 unique spacer sequences is used to classify $M t b$ strains based on their presence or absence. The patterns of presence and absence in each of the 43 spacer sequences can be summarized with a 43-digit binary code with 1 denoting the presence and 0 denoting the absence for each spacer, which can also be translated into a 15-digit numerical code [4] termed as the spoligotype.

\footnotetext{
* Correspondence: twee_hee_ong@nuhs.edu.sg

${ }^{2}$ Centre for Infectious Disease Epidemiology and Research, Saw Swee Hock School of Public Health, National University of Singapore, Singapore,

Singapore

Full list of author information is available at the end of the article
}

Spoligotypes can be used to compare $M t b$ isolates collected between different laboratories and countries. Spoligotyping is traditionally conducted using the PCRbased reverse line hybridization blotting technique [2]. Various new methods have recently been proposed for spoligotyping, the most of which are microarrays, such as the PixSysn QUAD 4500 Microarrayer [5], DNA microarray [6], hydrogel microarray (biochip) [7], Spoligorifytyping [8], and its follow-up TB-SPRINT [9]. Other spoligotyping methods include those based on a matrix-assisted laser desorption/ionization time-of-flight mass-spectrometry (MALDI-ToF MS) platform [10, 11]. Spoligotyping has also been applied to strain typing in other bacteria species such as Legionella pneumophila [12], Campylobacter jejuni [13, 14], and Salmonella [15].

Technological advancements in next-generation sequencing provide single nucleotide resolution for $M t b$ phylogenetic studies by allowing the construction of a single nucleotide polymorphism (SNP)-based phylogenetic tree. However, genotyping of bacteria is still needed for fast strain identification and correlation to previous isolates. For previous isolates, particularly the historical isolates, genotypes including spoligotypes may have been determined but whole genome sequences are not available and some isolates are not able to be sequenced. Under such circumstances, in silico genotyping from the whole genome sequences is necessary for correlating current isolates with previously genotyped ones. Several molecular genotyping techniques exist for $M t b$, of which 
the most widely used are: (1) spoligotyping; (2) mycobacterial interspersed repetitive units - variable numbers of tandem repeat (MIRU-VNTR); and (3) IS6110-based restriction fragment length polymorphism (IS6110RFLP) [16]. The inference of MIRU-VNTR from nextgeneration sequencing reads involves resolving the tandem repeats, which is extremely challenging for the current short sequence reads generated by the most widely used sequencing platforms. IS6110-RFLP commonly has its result based on the DNA fragment blots on electrophoresis gel image and thus focuses on the determination of the fragment lengths, which is also extremely challenging to infer since short read sequencing cannot be used alone to construct finished genomes. Spoligotyping, therefore, provides a unique opportunity to obtain the same result from whole genome sequences as the molecular genotyping result achieved in laboratories, which can correlate the isolates investigated using different approaches. In silico spoligotyping is also important for investigations using public data, where sequencing reads or complete genomic sequences are available but the spoligotypes of the isolates are not reported.

SpolPred [17] is a tool that accurately predicts the spoligotype of $M t b$ isolates from sequence reads of uniform length obtained from platforms such as Illumina GAII and HiSeq. However, for reads produced by platforms marketed for clinical diagnostics such as Illumina MiSeq and Ion sequencers, where the throughput is moderate and length of the reads are non-uniform, the accuracy of SpolPred is significantly reduced. SpoTyping improves the performance of SpolPred in three ways: (1) SpolPred reads in a fixed number of bases from each sequencing read as specified by the user. As a result, for sequencing experiments with non-uniform read length, the accuracy of prediction is highly dependent on the choices of the read length by the users. SpoTyping, by reading in the full length of the reads, makes use of all the available sequence data. (2) SpolPred requires the user to specify a direction for the reads, which can be either direct or reverse. However, since each FASTQ file consists of both direct and reverse reads, SpolPred only utilizes a fraction of the input sequence reads which can lead to incorrect predictions for sequencing experiments with low throughput. SpoTyping explicitly considers the reads in both directions, thereby using all the information presented in the sequence reads. (3) SpolPred relies on an inefficient sequence search algorithm, whereas SpoTyping integrates the BLAST algorithm in the search which reduces the time of the search considerably. In addition to the improvements listed above, SpoTyping also comes with novel functions not previously found in SpolPred or other software: (1) For TB disease outbreak investigation, it is necessary to quickly identify isolates with matching spoligotypes. SpoTyping thus automatically queries SITVIT [18], a global $M t b$ molecular markers database to retrieve associated epidemiological data for isolates with matched spoligotypes in an Excel spreadsheet, which can be presented as a graphical report showing the distribution summaries of the meta-data corresponding to the clades, years, and countries of isolation for these isolates. (2) SpoTyping works on different input files such as next-generations sequencing reads in FASTQ format, and complete genomic sequences or assembled contigs in FASTA format. (3) SpoTyping can be run on most operating systems such as Windows, Linux, and Mac OS, either as a non-interactive script which can be integrated into individual analysis pipelines or as an interactive application with a graphical user interface. Thus, we believe SpoTyping would be a useful tool for public health surveillance and genotyping from nextgeneration sequencing data in microbiological clinical diagnostic of $M t b$ strains.

\section{Implementation}

SpoTyping is implemented with Python and accepts two kinds of input files: single-end or pair-end sequence reads in FASTQ format, and complete genomic sequences or assembled contigs in FASTA format. A schematic representation of the SpoTyping workflow is shown in Fig. 1. When the input files are sequence reads, SpoTyping first concatenates all sequence reads in the input FASTQ file(s) into a single contiguous sequence in FASTA format which would be constructed into a BLAST [19] nucleotide database. The current program default (enabling the swift mode) is to read in no more than $250 \mathrm{Mbp}$ of the sequence reads, which corresponds to a read depth of approximately $55 \mathrm{X}$ of the $M t b$ genome and would be sufficient in most situations. Disabling the swift mode would require SpoTyping to utilize all sequence reads with increased execution time. The set of 43 spacer sequences, each of $25 \mathrm{bp}$ in length, would be queried against the constructed database using the standard nucleotide BLAST program. The BLAST output is then parsed to determine the number of hits for each spacer sequence in the input file(s). A maximum of one mismatch out of $25 \mathrm{bp}$ of the spacer sequence is allowed for a BLAST match to be considered as a hit. For sequence reads, if a spacer sequence is absent in the $M t b$ isolate, then no or very few hits would be identified, while if the number of hits exceeds a threshold (hit threshold has a default of five error-free hits and six 1-error-tolerant hits), it indicates the presence of the spacer sequence where the number of hits correlates with the sequence read depth of the locus. For genomic sequences or assembled contigs, the presence of one hit for a spacer sequence indicates the presence of the spacer. The binary string of 43 digits, each digit representing one of the 43 spacer sequences with 0 


\section{Query sequence 43 Spacers \\ Database

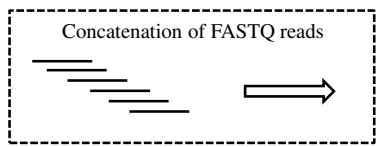 \\ Concatenated sequencing reads or genomic sequences in FASTA format \\ Result

\section{Query database SITVIT \\ Pie plot of the summary report}

A summary report for the query spoligotype offered by the database

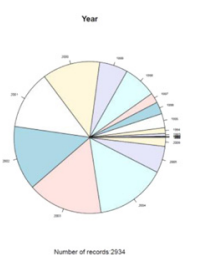

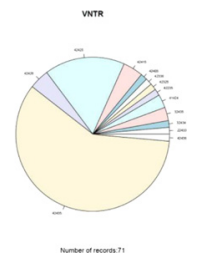

Fig. 1 A schematic representation of the SpoTyping workflow. If the specified input contains sequencing reads, SpoTyping first concatenates the sequencing reads to form an artificial sequence. The artificial sequence, or genetic sequences when the input contains complete genomic sequence or assembled contigs, would be built into the BLAST database. After querying the 43 spacer sequences in the database, the results are parsed to count the number of hits for each spacer sequence. A hit threshold is set to define a spacer as 'present' in the genome, resulting in a 43-digit binary code with 1 as present and 0 as absent, which is further translated into the octal code of the spoligotype. The SITVIT database is then queried to identify matching isolates having the same spoligotype, where the associated data of the matched isolates are downloaded and summarized as pie charts

indicating absence and 1 indicating presence, can therefore be written into an octal code that defines the spoligotype of the $M t b$ isolate. The predicted spoligotype is then automatically queried in the SITVIT database to retrieve all reported isolates having identical spoligotypes, where associated data corresponding to the MIRU12, VNTR, SIT, MIT, VIT, clades, countries of origin, countries of isolation, and year of report for these isolates would be downloaded in an Excel spreadsheet. SpoTyping also includes an $\mathrm{R}$ script that can present summary statistics of the associated meta-data as a pdf report.

The accuracy of SpoTyping was assessed in comparison with SpolPred on three datasets: (1) 161 isolates sequenced on Illumina HiSeq (SRA: SRA065095); (2) 30 isolates sequenced on Illumina MiSeq (ENA: PRJNA218508); and (3) 16 isolates sequenced on Ion Torrent (ENA: PRJEB6576). The first assessment was conducted on a dataset of $161 \mathrm{Mtb}$ isolates sequenced on Illumina HiSeq with experimentally determined spoligotypes reported [20]. Both SpoTyping and SpolPred were run with default parameters. The predicted octal codes were each queried in the SITVIT database to identify the matching spoligotype for comparison with the reported spoligotype. Discordant results were examined by searching the spacer sequences on the contigs assembled using the de novo assembly software Velvet [21]. The next assessment was conducted on a dataset of $30 \mathrm{Mtb}$ isolates sequenced on Illumina MiSeq without reported spoligotypes. The reference spoligotype for each isolate was determined by manual inspection of the BLAST output file to determine the number of hits for each spacer sequence in the sequence reads. Given that the sequence read depths are above 20X for all isolates, no hit for a spacer sequence is a strong indication of its absence while a number of above five hits is a strong indication of the presence of the spacer sequence. While a judgement cannot be safely made based on a hit number of 1 to 5 , isolates with at least one such case were removed from the study, leaving only isolates with confident reference spoligotypes. SpoTyping was run with default parameters while SpolPred calls for a specified read length, where a range of read lengths were used based on the read length percentiles from 0.04 to 1 at a step of 0.04 , resulting in a total of 25 predictions for each isolate. The accuracy of SpoTyping was also assessed in comparison with SpolPred on a dataset of $16 \mathrm{Mtb}$ isolates sequenced on Ion Torrent. The reference spoligotypes were determined similarly as those for Illumina MiSeq data. The running parameters were also similar as those for Illumina MiSeq data. 
The time performance of SpoTyping was compared with SpolPred based on the first dataset. The programs were run on a 64-bit Fedora Linux server workstation having a $2.0 \mathrm{GHz}$ quad processor and $32 \mathrm{~GB}$ RAM. Both SpoTyping and SpolPred were run twice for each isolate either with or without the swift mode. Default parameters were used for SpoTyping swift mode, while for nonswift mode, 10 error-free hits or 12 1-error-tolerant hits (options of -m 10 -r 12) was taken as the hit threshold due to the high sequencing coverage to eliminate false positives. For SpolPred, the pair-end sequence reads were first concatenated (concatenation time not counted toward the running time). The read lengths were set to be the actual read lengths. The hit threshold was similarly set to be 10 (option of $-\mathrm{m} 10$ ) in the non-swift mode.

The performance of SpoTyping was assessed for various sequence read depths to determine its applicable range, where we determined the accuracies of the SpoTyping prediction for: (1) an H37Ra Mtb isolate which had a sequencing throughput of 3,000 Mbp (approximately 670X); and (2) a Beijing-genotype $M t b$ isolate with a sequencing throughput of 2,700 Mbp (approximately 600X) by performing 50 iterations each for six down-sampling ratios of $50 \%, 20 \%, 10 \%, 5 \%$, $2 \%$, and $1 \%$ of the initial number of reads for each isolate. In each down-sampling experiment, a certain percent of the sequence reads were randomly selected from the original FASTQ file to form a new file with a lower read depth, where the percentage is called the down-sampling ratio. For all of the down-sampling experiments, default settings were used except for the categories of $2 \%$ and $1 \%$ where the hit threshold was set to two error-free hits and three 1-error-tolerant hits (options of $-\mathrm{m} 2-\mathrm{r} 3$ ). The false positives caused by the concatenation of sequence reads were also assessed in the down-sampling experiment.

The selection of the hit thresholds was also based on the down-sampling experiments. In each down-sampling experiment, the number of both error-free hits and 1error-tolerant hits for each spacer identified by SpoTyping were divided by the estimated read depth (number of sequence bases/ 4,500,000) of the experiment, representing the number of hits as a percentage of the estimated read depth. For each spacer in each experiment, the percentage is used as the feature to classify a spacer as present or absent, while the spacer's actual class of presence or absence is used to assess whether the classification is correct. A set of percentages was used as the thresholds to calculate the respective true positive rates and false positive rates, which were plotted as a receiver operating characteristic (ROC) curve. The thresholds were selected to maximize the true positive rate while minimizing the false positive rate.
The Beijing-genotype isolate can be accessed through the European Nucleotide Archive (ENA) code ERP006354. The $\mathrm{H} 37 \mathrm{Ra}$ isolate is a laboratory strain that was sequenced as part of a validation sequencing run, and the FASTQ files will be provided upon request.

\section{Results \\ In silico spoligotyping of $161 \mathrm{Mtb}$ isolates sequenced on Illumina HiSeq}

For all the $161 \mathrm{Mtb}$ isolates, SpoTyping and SpolPred predicted the same spoligotypes (Additional file 1: Table S1), of which 20 isolates either without a match in the SITVIT database or reported as 'New' were excluded from subsequent comparisons. Of the remaining 141 isolates, predictions of SpoTyping and the laboratory determined spoligotypes for 127 isolates (90.07\%) were identical. For the 14 discordant isolates, the spacer sequences were searched in the assembled contigs to determine the spoligotypes, which are all concordant with the predictions from SpoTyping (Additional file 1: Table S2).

\section{In silico spoligotyping of $30 \mathrm{Mtb}$ isolates sequenced on Illumina MiSeq}

The accuracy of SpoTyping was then assessed in comparison with SpolPred on $30 \mathrm{Mtb}$ isolates sequenced on Illumina MiSeq, among which 21 passed filtering for having reference spoligotypes confidently determined. SpoTyping correctly inferred the spoligotypes for all 21 isolates. Since SpolPred requires for a read length to be specified as input, a range of read lengths were assessed based on the percentiles from 0.04 to 1 at a step of 0.04 , resulting in a total of 25 predictions for each isolate whose read length specifications are summarized in Additional file 1: Table S3. At each percentile, the predictions for the 21 isolates were analyzed to calculate the prediction accuracy, which is summarized in Fig. 2 and Additional file 1: Table S4. SpolPred performs the best using the read lengths at the $0.36,0.40$, or 0.44 percentiles, with accuracies around $50 \%$. The prediction accuracy of SpolPred is significantly lower than that obtained by SpoTyping and is also highly dependent on the choice of read length used as input which in itself is difficult to determine.

\section{In silico spoligotyping of $16 \mathrm{Mtb}$ isolates sequenced on Ion Torrent}

The accuracy for spoligotype inference was also determined on $16 \mathrm{Mtb}$ isolates sequenced on Ion Torrent with spoligotypes reported to be all Beijing genotype [22]. Of the 16 isolates, 11 have confidently determined spoligotypes, which are all of the spoligotype '000000000003771' as are consistent with the reported Beijing genotype. SpoTyping makes correct prediction for all the 11 isolates. The performance of SpolPred is 

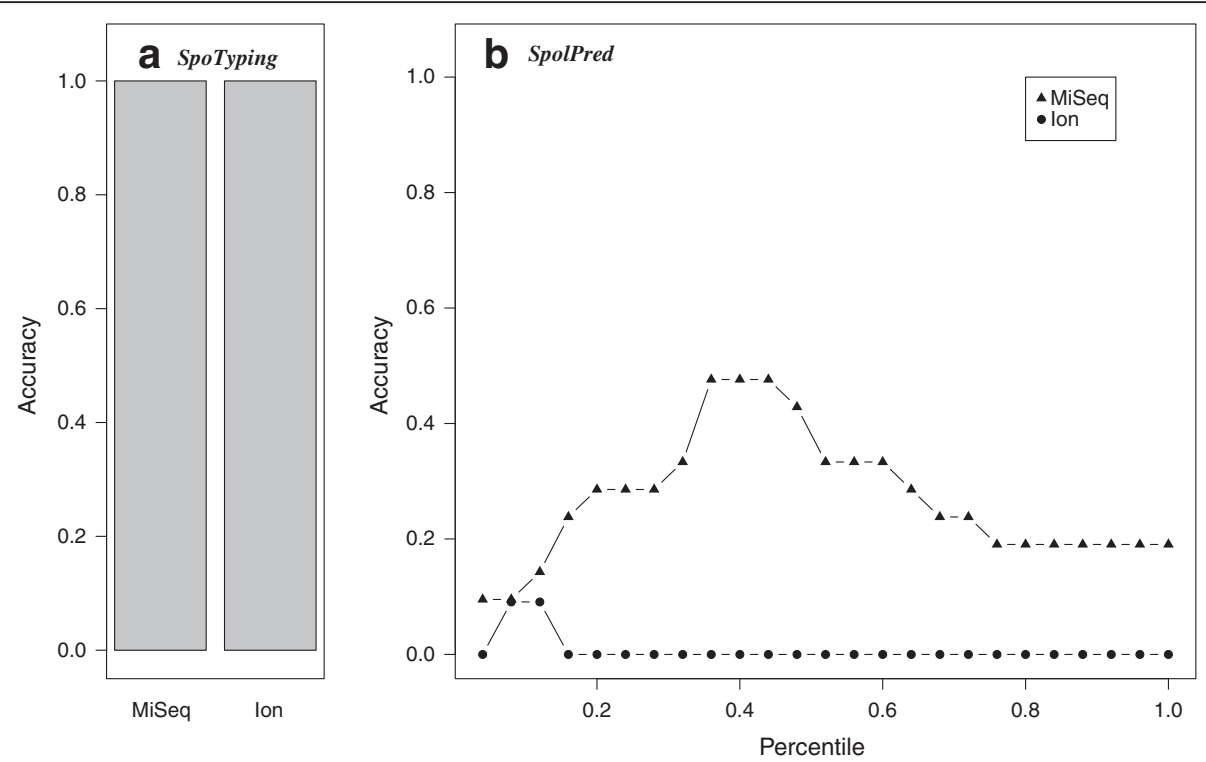

Fig. 2 Prediction accuracy of Mtb isolates sequenced on Illumina MiSeq and Ion Torrent. SpolPred requires a read length to be specified which results in inconsistent predictions for different specifications. The accuracy assessment was conducted between SpoTyping (a) and SpolPred (b) on 21 MiSeq-sequenced isolates and 11 lon-sequenced isolates, with SpoTyping predictions using default parameters and SpolPred predictions using different read length percentiles as the input read lengths. While SpoTyping have perfect accuracies for both datasets, SpolPred gives varying accuracies depending on the read length, which are always lower than $50 \%$

summarized in Fig. 2, Additional file 1: Table S5 and Table S6. SpolPred performs best using the read length at the 0.08 and 0.12 percentile, with accuracies of only around $10 \%$.

\section{Comparison of time performance for SpoTyping and SpolPred on $161 \mathrm{Mtb}$ isolates}

For the $161 \mathrm{Mtb}$ isolates tested, SpoTyping is about 20 to 40 times faster than SpolPred, with SpoTyping taking an average of $28.8 \mathrm{~s}$ (standard deviation is $5.3 \mathrm{~s}$ ) in its swift mode, and an average of $56.4 \mathrm{~s}$ (standard deviation is $8.0 \mathrm{~s}$ ) to process all reads, while SpolPred took an average of $17 \mathrm{~min} 19.3 \mathrm{~s}$ (standard deviation is $1 \mathrm{~min}$ $35.3 \mathrm{~s}$ ) by using the $-\mathrm{s}$ option, or an average of $18 \mathrm{~min}$ $20.0 \mathrm{~s}$ (standard deviation is $50.2 \mathrm{~s}$ ) to process all reads (Additional file 1: Table S1).

\section{Down-sampling experiments}

Based on the down-sampling experiments which first explore the applicable throughput for accurate spoligotype inference, SpoTyping is able to efficiently and accurately predict the spoligotype for isolates having sequencing throughput over $54 \mathrm{Mbp}$ (read depth of approximately $12 \mathrm{X}$ ) with accuracies above $98 \%$ (Fig. 3, Additional file 1: Table S7 for H37Ra and Additional file 1: Table S8 for Beijing). However, for isolates that are sequenced at very low coverage (below 10X), using the lower threshold is still not sufficient to make accurate predictions as some of the spacer sequences would not be adequately sequenced and represented in the input FASTQ file(s).

Since SpoTyping concatenates sequence reads into an artificial sequence to create the BLAST database, an immediate concern is the false positives created due to chimera sequences. In all of 600 down-sampling experiments performed for both H37Ra and Beijing genotype $M t b$ isolates, the maximum number of false positive hit is 1 for both error-free hits and 1-error-tolerant hits. Of the experiments, $98.3 \%(590 / 600)$ show no false positive error-free hits while $95.7 \%(574 / 600)$ show no false positive 1-error-tolerant hits. The likelihood of false positives created due to chimera sequences is thus low which can be further reduced by setting more stringent hit thresholds.

\section{Threshold selection}

We evaluated the choice of the hit thresholds to determine the presence or absence of a spacer sequence used in SpoTyping. The evaluation was conducted in the down-sampling experiments, based on the groups with down-sampling ratios from $2 \%$ to $50 \%$ (read depths between approximately $12 \mathrm{X}$ and approximately 300X) where accurate inferences for the spacer sequences are possible to be made. A total of 21,586 spacer sequence instances ((5 down-sampling ratios * 50 rounds for each down-sampling ratio * 43 spacer for each round +43 spacers without down-sampling) $=10,793$ spacers for each of the two strains) with their respective number of 


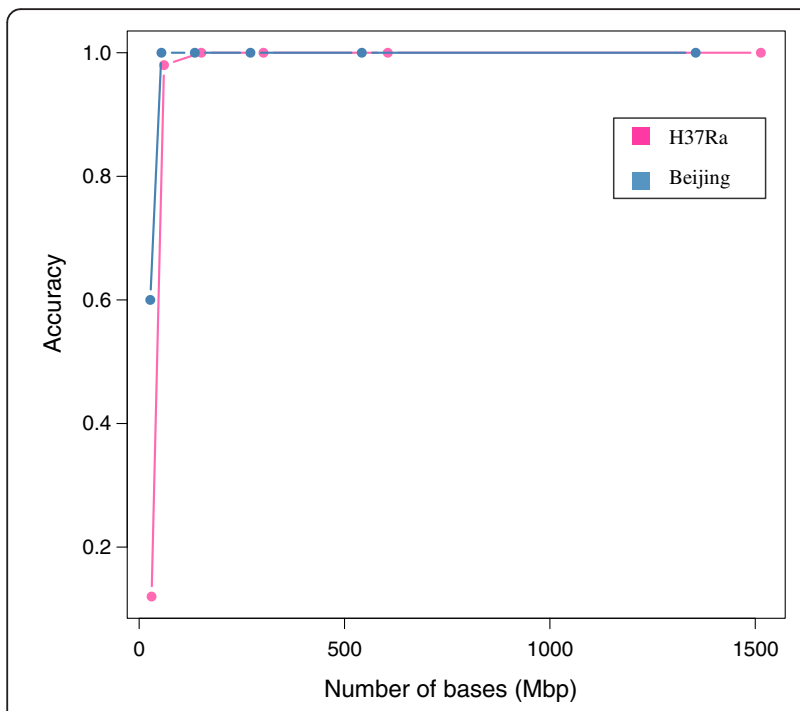

Fig. 3 Assessing the accuracy of SpoTyping across various sequence read depths for H37Ra and Beijing-genotype isolates. With blue points denoting the Beijing genotype, pink points denoting H37Ra, the prediction accuracies were assessed with the sequencing throughput measured by the number of bases for all the downsampling experiments. SpoTyping is suitable for sequencing runs with throughput over $54 \mathrm{Mbp}$ (estimated depth of approximately 12X), where the accuracy is almost $100 \%$

hits identified by SpoTyping were included in the analysis, of which 10,040 are absent cases and 11,546 are present cases. The number of hits was divided by the estimated read depth to represent the number of hits as a percentage of the read depth in order to adjust for the difference in sequencing throughput. A set of percentages was used as the thresholds to calculate the respective true positive rates and false positive rates, which were plotted as an ROC curve (Fig. 4). The ROC curves for both the error-free hits (Fig. 4a) and 1-error-tolerant hits (Fig. 4b) show very high true positive rates and very low false positive rates, with the areas under the ROC being 0.9999997 and 0.9999998, respectively. False positive rates are always nearly 0 , while the true positive rates are above $99 \%$ by setting the thresholds to be $1.80 \%$ to $14.86 \%$ of the read depth for error-free hits and $1.80 \%$ to 14.88 $\%$ of the read depth for 1 -error-tolerant hits. Thus the default thresholds of five error-free hits and six 1error-tolerant hits are applicable to sequencing experiments with estimated read depths between approximately 30X and approximately 280X. The thresholds can be adjusted accordingly given sequencing throughputs beyond this range.

\section{Discussion}

The increasing global burden of $\mathrm{TB}$, especially drugresistant strains, has put a significant spotlight on pathogen whole genome sequencing as a rapid diagnostic tool, which is of great relevance to both public health surveillance and clinical treatment. The application of next-generation sequencing in clinical microbiology requires fast and easy-to-use software that is able to accurately produce easily comprehensible results. As shown, SpoTyping is able to accurately determine the spoligotype of the $M t b$ isolate rapidly. Contrary to SpolPred which is sensitive to the user-specified read length and gives inconsistent predictions at different
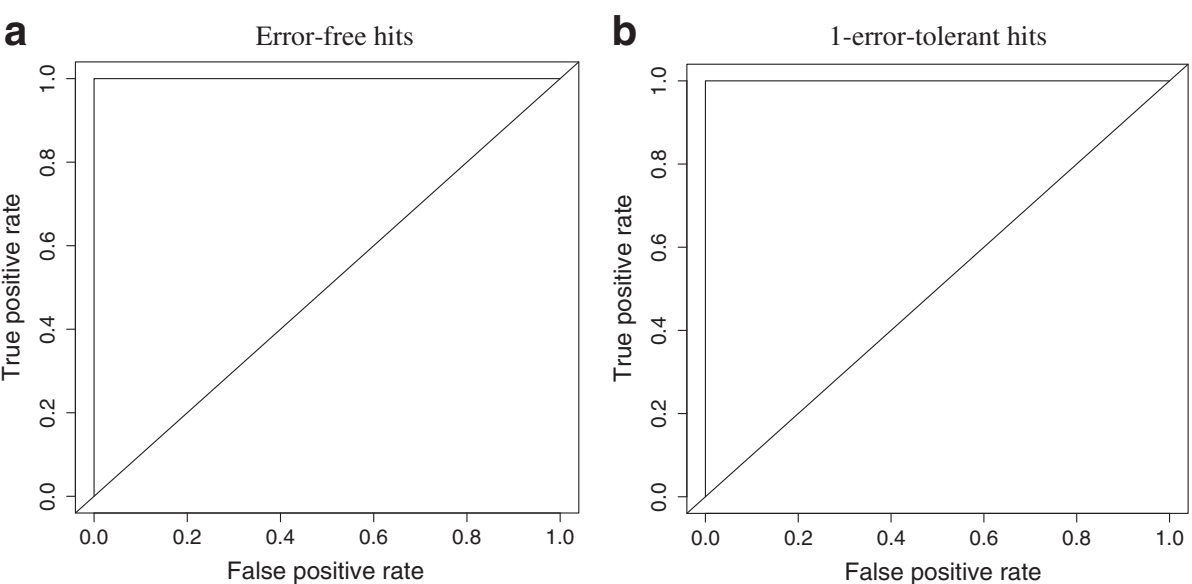

Fig. 4 ROC curves for the selection of hit thresholds. The ROC curves were plotted for both error-free hits (a) and 1-error-tolerant hits (b) to select the hit thresholds. Diagonal lines, also known as lines of no discrimination, were plotted as references of random guess. The threshold evaluation was based on a percentage calculated as the number of hits divided by the estimated read depth. A set of percentages was used as the thresholds to calculate the respective true positive rates and false positive rates, which were plotted as the ROC curves. Both ROC curves show constantly high true positive rates and low false positive rates, with the areas under the ROC being 0.9999997 and 0.9999998 , respectively 
read lengths, SpoTyping gives accurate predictions based on sequence reads produced from different technologies regardless of the length uniformity of the sequence reads and is 20 to 40 times faster than SpolPred. The additional functions of database query, information visualization and report generation provided by SpoTyping where the predicted spoligotype is automatically queried in the SITVIT database to retrieve all associated epidemiological data corresponding to the MIRU12, VNTR, SIT, MIT, VIT, clades, countries of origin, countries of isolation, and year of report and presented as a report would be a useful tool for public health surveillance of $M t b$ strains causing tuberculosis.

While there are several molecular typing techniques for $M t b$, the most widely used are spoligotyping, MIRUVNTR, and IS6110-RFLP. Spoligotyping, though being a relatively simple, cost-effective, and high-throughput method, suffers from the limitations of: (1) having relatively low discriminatory power [23] due to its use of only a single genetic locus; and (2) having limited use in phylogenetic study. Among the genotyping methods for $M t b$, a combination of spoligotyping and MIRU-VNTR was reported to be the best strategy [24, 25]. However, significant technical challenges currently exist for the accurate in silico typing from next-generation sequencing reads of MIRU-VNTR which involves resolving tandem repeats and IS6110-RFLP whose result is based on DNA fragment blots on electrophoresis gel image and thus involves the determination of the fragment lengths. Spoligotyping, as a result, provides a unique chance to obtain the same result from whole genome sequences as the molecular typing result achieved in laboratories, which can correlate the isolates investigated with different approaches. Though spoligotyping has less discrimination power than SNP phylogeny inferred from whole genome sequences, it is unique in correlating the genomic data produced in research labs and the molecular typing data from clinical laboratories. Thus in silico spoligotyping is not only a genotyping method for $M t b$ isolate differentiation, but also a bridge between isolates with whole genome sequences available and isolates typed and investigated with traditional laboratory protocols, especially those historical isolates that are not sequenced. Inexorably, clinical surveillance and management of TB, particularly for disease diagnosis and treatment, will progress towards the use of direct $M t b$ sequencing. Thus the ease of use and interpretability of the results will be of considerable importance to users within a clinical setting, which is well achieved with SpoTyping.

A recently published letter reported CASTB, an analysis server for the Mycobacterium tuberculosis complex, which provides next-generation sequencing data analysis tools for virtual typing (spoligotyping included), virtual drug resistance analysis, and phylogenetic analysis [26]. While the webserver provides a comprehensive overview at the sequencing data, the performance of each tool is not well evaluated in the publication. More accurate and well assessed tools are thus needed for further analysis. SpoTyping is well assessed to provide high accuracy for in silico spoligotyping and thus demonstrates the reliability of the results. SpoTyping also benefits from its open source nature that it can be easily integrated into in-house analysis pipelines for in-depth analysis of the sequencing data. When talking about execution time, services provided by web servers may be very slow due to the inherent issues such as the process of data uploading and the availability of the computational resources. SpoTyping, on the other hand, can be set up locally and provides the spoligotyping result within a minute.

For the 14 discordant spoligotypes between the laboratory tests and the in silico predictions made by SpoTyping in the $161 \mathrm{Mtb}$ isolates sequenced on Illumina HiSeq, the SNP-based phylogenetic tree of these $161 \mathrm{Mtb}$ isolates in the original article [20] was examined to compare the lineage with the spoligotyping results (Additional file 1: Table S9). Out of the 14 discordant results, three showed better concordance of the in silico prediction with the lineage on the tree. As an example, an isolate (Accession: SRR671868, Strain: 143) located at Lineage 4.2 on the SNP-based phylogenetic tree is reported to be a Beijing genotype based on the laboratory test in the publication, while predicted to be a T2 genotype by SpoTyping. However, the Beijing genotype is usually found at East Asia Lineage 2, while Lineage 4 usually harbors the Euro-American genotypes. One of the discrepancies may be caused by the different naming of spoligotypes in different databases (Beijing and Beijinglike). Definite conclusion cannot be made for the remaining 10 isolates for which the reported spoligotype and in silico predicted spoligotype are different while the lineages for both spoligotypes are similar (T2 and H3, for example). For such isolates, the difference could be due to the discrepancy between laboratory tests and the genomic features.

\section{Conclusions}

SpoTyping is an accurate, fast, and easy-to-use program for in silico spoligotyping of $M t b$ isolates from nextgeneration sequencing reads, complete genomic sequences, and assembled contigs. In addition, SpoTyping automatically queries the global $M t b$ molecular markers database SITVIT to retrieve associated data for matching isolates with the inferred spoligotypes, which can be summarized graphically to generate a report. SpoTyping would be a useful tool for public health surveillance and genotyping of $M t b$ strains. 


\section{Availability and requirements}

- Project name: SpoTyping

- Project home page: https://github.com/xiaeryu/ SpoTyping-v2.0

- Operating systems: Linux, Mac OS, Windows

- Programming language: Python (version 2.7)

- Other requirements: BLAST

- License: GNU General Public License

- Any restrictions to use by non-academics: None

\section{Additional file}

Additional file 1: Table S1. Spoligotype prediction and time performance of 161 Mtb isolates with SpoTyping in comparison with SpolPred. Table S2. Spoligotypes of 14 Mtb isolates determined from contigs obtained by de novo assembly, Velvet. Table S3. Actual length at different percentiles used as the read length for SpolPred spoligotype prediction of isolates sequenced on Illumina MiSeq. Table S4. Spoligotype prediction for Mtb isolates sequenced by Illumina MiSeq with SpoTyping in comparison with SpolPred. Table S5. Actual length at different percentiles used as the read length for SpolPred spoligotype prediction of isolates sequenced on Ion Torrent. Table S6. Spoligotype prediction for Mtb isolates sequenced by lon Torrent with SpoTyping in comparison with SpolPred. Table S7. Statistics of time and accuracy of running SpoTyping on 50 iterations each for various down-sampling ratios of an H37Ra Mtb isolate. Table S8. Statistics of time and accuracy of running SpoTyping on 50 iterations each for various down-sampling ratios of a Beijing Mtb isolate. Table S9. Lineages of 14 discordant Mtb isolates by SpoTyping, experimentally reported and phylogenetic tree. (XLSX 55 kb)

\section{Abbreviations}

CRISPR: clustered regularly interspaced short palindromic repeats; DR: direct repeat; MIRU-VNTR: mycobacterial interspersed repetitive units - variable numbers of tandem repeat; Mtb: Mycobacterium tuberculosis; RFLP: restriction fragment length polymorphism; ROC: receiver operating characteristic; SNP: single nucleotide polymorphism; TB: tuberculosis.

\section{Competing interests}

The authors declare that they have no competing interests.

\section{Authors' contributions}

EX, YYT, and RTHO conceived the project. EX designed and implemented the project with help from YYT and RTHO. EX and RTHO wrote the manuscript. All authors read and approved the final manuscript.

\section{Acknowledgements}

The authors thank Li-Hwei Sng and Justine Peh from Central Tuberculosis Laboratory, Department of Pathology, Singapore General Hospital for the culture and DNA extraction of the H37Ra and Beijing genotype Mtb isolates, and Christopher Wong, Min Gong, and Hui-Maan Seah from Genome Institute of Singapore for the whole genome sequencing of both H37Ra and Beijing genotype Mtb isolates.

\section{Funding}

EX is supported by a scholarship from NUS Graduate School for Integrative Sciences and Engineering.

\section{Author details}

${ }^{1}$ NUS Graduate School for Integrative Sciences and Engineering, National University of Singapore, Singapore, Singapore. ${ }^{2}$ Centre for Infectious Disease Epidemiology and Research, Saw Swee Hock School of Public Health, National University of Singapore, Singapore, Singapore. ${ }^{3}$ Department of Statistics and Applied Probability, National University of Singapore, Singapore, Singapore. ${ }^{4}$ Life Sciences Institute, National University of
Singapore, Singapore, Singapore. ${ }^{5}$ Genome Institute of Singapore, Singapore, Singapore.

Received: 21 October 2015 Accepted: 25 January 2016

Published online: 17 February 2016

\section{References}

1. World Health Organization. Tuberculosis Fact Sheet 104. Geneva: WHO; 2015

2. van der Zanden AG, Hoentjen AH, Heilmann FG, Weltevreden EF, Schouls LM, van Embden JD. Simultaneous detection and strain differentiation of Mycobacterium tuberculosis complex in paraffin wax embedded tissues and in stained microscopic preparations. Mol Pathol. 1998:51:209-14.

3. Hermans PWM, Van Soolingen D, Bik EM, De Haas PEW, Dale JW, Van Embden JDA. Insertion element IS987 from Mycobacterium bovis BCG is located in a hot-spot integration region for insertion elements in Mycobacterium tuberculosis complex strains. Infect Immun. 1991;59:2695-705.

4. Dale JW, Brittain D, Cataldi AA, Cousins D, Crawford JT, Driscoll J, et al. Spacer oligonucleotide typing of bacteria of the Mycobacterium tuberculosis complex: Recommendations for standardised nomenclature. Int J Tuberc Lung Dis. 2001;5(3):216-9.

5. Song EJ, Jeong HJ, Lee SM, Kim CM, Song ES, Park YK, et al. A DNA chipbased spoligotyping method for the strain identification of Mycobacterium tuberculosis isolates. J Microbiol Methods. 2007:68:430-3.

6. Ruettger A, Nieter J, Skrypnyk A, Engelmann I, Ziegler A, Moser I, et al. Rapid spoligotyping of Mycobacterium tuberculosis complex bacteria by use of a microarray system with automatic data processing and assignment. J Clin Microbiol. 2012;50:2492-5.

7. Bespyatykh JA, Zimenkov DV, Shitikov EA, Kulagina EV, Lapa SA, Gryadunov DA et al. Spoligotyping of Mycobacterium tuberculosis complex isolates using hydrogel oligonucleotide microarrays. Infect Genet Evol. 2014;26:41-6.

8. Gomgnimbou MK, Abadia E, Zhang J, Refrégier G, Panaiotov S, Bachiyska E, et al. "Spoligoriftyping", a dual-priming-oligonucleotide-based directhybridization assay for tuberculosis control with a multianalyte microbeadbased hybridization system. J Clin Microbiol. 2012;50:3172-9.

9. Gomgnimbou MK, Hernández-Neuta I, Panaiotov S, Bachiyska E, Palomino JC, Martin A, et al. Tuberculosis-spoligo-rifampin-isoniazid typing: an all-in-one assay technique for surveillance and control of multidrug-resistant tuberculosis on Luminex devices. J Clin Microbiol. 2013;51:3527-34.

10. Honisch C, Mosko M, Arnold C, Gharbia SE, Diel R, Niemann S. Replacing reverse line blot hybridization spoligotyping of the Mycobacterium tuberculosis complex. J Clin Microbiol. 2010;48:1520-6.

11. Shitikov E, llina E, Chernousova L, Borovskaya A, Rukin I, Afanas'ev M, et al. Mass spectrometry based methods for the discrimination and typing of mycobacteria. Infect Genet Evol. 2012;12:838-45.

12. Ginevra C, Jacotin N, Diancourt L, Guigon G, Arquilliere R, Meugnier H, et al. Legionella pneumophila sequence type 1/Paris pulsotype subtyping by spoligotyping. J Clin Microbiol. 2012;50:696-701.

13. Price EP, Smith H, Huygens F, Giffard PM. High-resolution DNA melt curve analysis of the clustered, regularly interspaced short-palindromic-repeat locus of Campylobacter jejuni. Appl Environ Microbiol. 2007;73:3431-6.

14. Schouls LM, Reulen S, Duim B, Wagenaar JA, Willems RJL, Dingle KE, et al. Comparative genotyping of Campylobacter jejuni by amplified fragment length polymorphism, multilocus sequence typing, and short repeat sequencing: strain diversity, host range, and recombination. J Clin Microbiol. 2003:41:15-26.

15. Fabre L, Zhang J, Guigon G, Le Hello S, Guibert V, Accou-Demartin M, et al. CRISPR typing and subtyping for improved laboratory surveillance of Salmonella infections. PLoS One. 2012;7:e36995.

16. Barnes PF, Cave MD. Molecular epidemiology of tuberculosis. N Engl J Med. 2003:349:1149-56.

17. Coll F, Mallard K, Preston MD, Bentley S, Parkhill J, McNerney R, et al. SpolPred: rapid and accurate prediction of Mycobacterium tuberculosis spoligotypes from short genomic sequences. Bioinformatics. 2012;28(22):2991-3.

18. Demay $C$, Liens $B$, Burguière $T$, Hill V, Couvin D, Millet J, et al. SITVITWEB - A publicly available international multimarker database for studying Mycobacterium tuberculosis genetic diversity and molecular epidemiology. Infect Genet Evol. 2012;12:755-66.

19. Altschul SF, Gish W, Miller W, Myers EW, Lipman DJ. Basic local alignment search tool. J Mol Biol. 1990;215(3):403-10. 
20. Zhang H, Li D, Zhao L, Fleming J, Lin N, Wang T, et al. Genome sequencing of 161 Mycobacterium tuberculosis isolates from China identifies genes and intergenic regions associated with drug resistance. Nat Genet. 2013:45:1255-60.

21. Zerbino DR, Birney E. Velvet: Algorithms for de novo short read assembly using de Bruijn graphs. Genome Res. 2008;18:821-9.

22. Witney AA, Gould KA, Arnold A, Coleman D, Delgado R, Dhillon J, et al. Clinical application of whole genome sequencing to inform treatment for multi-drug resistant tuberculosis cases. J Clin Microbiol. 2015;53(5):1473-83.

23. Roetzer A, Schuback S, Diel R, Gasau F, Ubben T, Di Nauta A, et al. Evaluation of Mycobacterium tuberculosis typing methods in a 4-year study in SchleswigHolstein, Northern Germany. J Clin Microbiol. 2011;49:4173-8.

24. Oelemann MC, Diel R, Vatin V, Haas W, Rüsch-Gerdes S, Locht C, et al. Assessment of an optimized mycobacterial interspersed repetitive-unitvariable-number tandem-repeat typing system combined with spoligotyping for population-based molecular epidemiology studies of tuberculosis. J Clin Microbiol. 2007:45:691-7.

25. Allix-Béguec C, Fauville-Dufaux M, Supply P. Three-year population-based evaluation of standardized mycobacterial interspersed repetitive-unitvariable-number tandem-repeat typing of Mycobacterium tuberculosis. J Clin Microbiol. 2008;46:1398-406.

26. Iwai H, Kato-miyazawa M, Kirikae T, Miyoshi-akiyama T. CASTB (the comprehensive analysis server for the Mycobacterium tuberculosis complex): A publicly accessible web server for epidemiological analyses, drug-resistance prediction and phylogenetic comparison of clinical isolates. Tuberculosis. 2015;95(6):843-4.

\section{Submit your next manuscript to BioMed Central and we will help you at every step:}

- We accept pre-submission inquiries

- Our selector tool helps you to find the most relevant journal

- We provide round the clock customer support

- Convenient online submission

- Thorough peer review

- Inclusion in PubMed and all major indexing services

- Maximum visibility for your research

Submit your manuscript at www.biomedcentral.com/submit

) Biomed Central 